\title{
MACHO PHOTOMETRY AND ASTROMETRY
}

\author{
E. HØG AND I.D. NOVIKOV
}

Copenhagen University Observatory - NBIfAFG

Østervoldgd. 3, DK-1350 Copenhagen K, Denmark

AND

A.G. POLNAREV

Astronomy Unit, Queen Mary and Westfield College

University of London, Mile End Road, E1 4NS London, UK

\begin{abstract}
MACHOs (Massive Astrophysical Compact Halo Objects) have been discovered by their relativistic amplification of light from distant stars as they crossed very near to the line-of-sight. The very few events were detected from more than a billion photometric measurements of millions of stars in the LMC. - A mathematical theory of analysis of astrometric and photometric measurements of microlensing events is presented. It is shown that three photometric measurements and three one-dimensional astrometric measurements during an event are, in principle, sufficient to determine the - precisely - six observable physical parameters of the MA$\mathrm{CHO}$, including the proper motion and distance of the dark body, provided the position, motion and distance of the undeflected star has been determined from observations outside the event. The practical possibility of such observations is discussed by comparison with the proposed ROEMER satellite, an instrument concept with the potential for obtaining the many billions of photometric and astrometric observations of the required quality. At least 300 photometric amplifications of light from stars brighter than $V=16.5$ mag could de detected during a ROEMER mission, but the prospects for significant astrometric observations are meagre.
\end{abstract}

\section{References}

Høg E., Novikov I.D., Polnarev A.G. 1994, MACHO Photometry and Astrometry, Astron.\& Astrophys. (in press) 